\title{
Applications of Aberration Corrected TEM and Exit Wavefunction Reconstruction to Materials Science.
}

Angus Kirkland ${ }^{1}$, Judy Kim ${ }^{1}$, Jamie Warner ${ }^{1}$, Konstantin Borisenko ${ }^{1}$ Sarah Haigh $^{2}$, Neil Young ${ }^{1}$, Peng Wang $^{3}$ and Peter Nellist ${ }^{1}$

1. University of Oxford, Department of Materials, Parks Road, Oxford OX13PH, UK.

2. School of Materials, The University of Manchester, Manchester M13 9PL, UK.

3. National Laboratory of Solid State Microstructures and College of Engineering and Applied sciences, Nanjing University, Nanjing 210093, People's Republic of China.

The initial announcement of two successful alternative geometries of successful aberration correctors for TEM [1] and STEM [2] in 1997 has led to significant improvements in spatial resolution and the availability of higher probe current densities for analysis. There are now in excess of 400 aberration correctors installed worldwide. The Oxford-JEOL 2200MCO was the first instrument fitted with two aberration correctors, installed in 2003 [3] and this instrument has more recently been upgraded with a monochromated source [4].

In this paper I will highlight three areas of research carried out in Oxford since the installation of the instrument above that have been enabled by aberration correction.

The enhanced resolution provided by aberration correction is particularly important at low accelerating voltages when combined with low energy width sources. It is now possible to record atomic resolution data from radiation sensitive materials including Graphene and its derivatives (Figure 1) [5]. I will briefly discuss the use of aberration corrected imaging for the study of point and line defects, their evolution and mobility in this important class of material.

Aberration correction has also enabled novel experimental geometries including Scanning Confocal EM (SCEM) [6] that are not usefully available in uncorrected instruments. In the SCEM configuration the pre-specimen optics are the same as in STEM and the post-specimen optics are used to image electrons that have been scattered from the confocal point onto a aperture in the detector plane. Electrons scattered from elsewhere are focused to a point either above or below the aperture such that their contribution to the image intensity is reduced. In this way inelastic SCEM or EFSCEM gives improved depth selectivity, allowing 3-D structure determination, which will be discussed.

Finally, aberration correction has benefitted indirect exit wavefunction restoration [7], in particular from datasets consisting of a series of images recorded with varying illumination tilt where the limiting, critical conditioning between the defocus and tilt angle is relaxed. Subsequently, larger tilt angles can be used in the dataset for restoration leading to higher resolution in the recovered wavefunction (Figure 2). This approach has shown that it is possible to extend the interpretable resolution of the latest generation of aberration corrected instruments by $41 \%$ relative to that which is achievable using axial imaging. However, the technique has limitations and constraints and these will be discussed together with possible alternative geometries for dataset acquisition. 

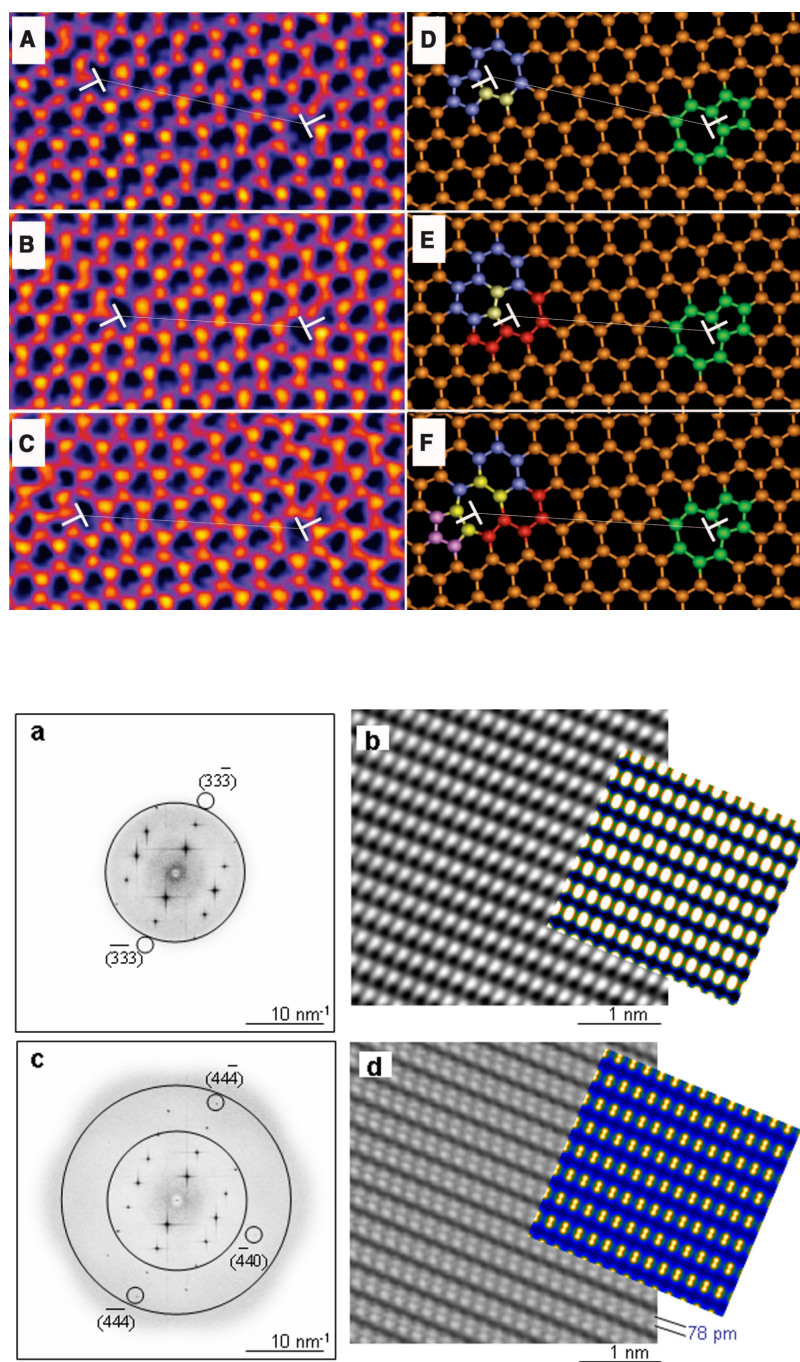

Figure 1. Real-time dislocation dynamics. HRTEM images showing changes in the position of an edge glide dislocation with time under continuous electron beam irradiation. (a) Time = 0 s. (b) Time $=141$ s. (c) Time $=321$ s. (d-f) Atomic models illustrating the structures inferred from (a) to (c), respectively. The white " $\mathrm{T}$ " indicates the position of the dislocation.

Figure 2. a) Restored exit wavefunctions for $<112>$ oriented silicon. Moduli of the complex Fourier transforms of the exit wave-function restored from focal and tilt-defocus series are shown in (a) and (c) respectively. Contrast is inverted to improve clarity. The $10 \%$ axial information limit corresponding to a real space resolution of $0.117 \mathrm{~nm}$ is indicated on both (a) and (c). In (c) the 10\% information limit for the tilt-defocus data set in the direction of weakest transfer (corresponding to $0.071 \mathrm{~nm}$ resolution) is indicated by the larger circle. Sub-regions of the real space phase of the specimen exit wavefunction restored from focal series and from the tilt-defocus data set are shown in (b) and (d) respectively with simulated wavefunctions overlaid.

\section{References:}

[1] M. Haider et al., Nature 392 (1998) p.768.

[2] O.L. Krivanek, N. Dellby and A.R. Lupini, Ultramicroscopy 78

(1999) p.1.

[3] H. Sawada et al., J. Electron Microsc (Tokyo) 54 (2005) p.119.

[4] M. Mukai et al., Ultramicroscopy, in press (2014).

[5] J. H. Warner et al., Science 337 (2012) p.209.

[6] E.C. Cosgriff et al., Advances in Imaging and Electron Physics, Ed. P W Hawkes 162 (2010) p.45

[7] S. Haigh, H. Sawada, A. I. Kirkland, Phil. Trans. Proc. Roy. Soc. A367 (2009) p.3755.

[8] Financial support from EPSRC (EP/F048009/1) and (EP/K032518/1) is gratefully acknowledged. 